\title{
Superfluid rotation sensor with helical laser trap.
}

\author{
A.Yu.Okulov* \\ Russian Academy of Sciences, 119991, Moscow, Russia
}

(Dated: November 12, 2012)

\begin{abstract}
The macroscopic quantum states of the dilute bosonic ensemble in helical laser trap at the temperatures about $10^{-6} \mathbf{K}$ are considered in the framework of the Gross-Pitaevskii equation. The helical interference pattern is composed of the two counter propagating Laguerre-Gaussian optical vortices with opposite orbital angular momenta $\ell \hbar$ and this pattern is driven in rotation via angular Doppler effect. Macroscopic observables including linear momentum and angular momentum of the atomic cloud are evaluated explicitly. It is shown that rotation of reference frame is transformed into translational motion of the twisted matter wave. The speed of translation equals the group velocity of twisted wavetrain $V_{z}=\Omega \ell / k$ and alternates with a sign of the frame angular velocity $\Omega$ and helical pattern handedness $\ell$. We address detection of this effect using currently accessible laboratory equipment with emphasis on the difference between quantum and classical fluids.
\end{abstract}

PACS numbers: $37.10 . \mathrm{Gh}$ 42.50.Tx 67.85.Hj 42.65.Hw

\section{INTRODUCTION}

The superfluids are often considered as an absolute reference frame. This means that when a density of condensate fraction is large enough $\rho_{s} \rightarrow \rho$ the quantum fluid confined in annular container (fig.1a) and cooled below phase transition temperature $T_{\lambda}$ proves to be in irrotational state with zero angular momentum $L_{z}=0$ regardless to the changes of container's angular velocity $\Omega\left[1\right.$. This happens at rather slow rotation rates $\left(\Omega / 2 \pi \leq \cdot 10^{-3} H z\right)$. At a higher angular velocities a metastable persistent flow with nonzero angular momentum per particle $L_{z}=\ell \hbar$ is observable [2]. The same holds for the quantum gas in multiply connected optical and magnetic traps [3, 4], where order parameter $\Psi$ is given by the Gross-Pitaevskii equation (GPE) [5]:

$$
i \hbar \frac{\partial \Psi}{\partial t}=-\frac{\hbar^{2}}{2 m} \Delta \Psi+U_{e x t}(z, r, \theta, t) \Psi+g|\Psi|^{2} \Psi
$$

where $m$ is atomic mass, $g=4 \pi \hbar^{2} a_{S} / m$ is interaction constant, $a_{S}$ is S-wave scattering length, $U_{\text {ext }}$ is external confining potential, $(z, r, \theta)$ are cylindrical coordinates. For the quantum liquid confined by an annular container (fig.1a) with impenetrable walls the potential is a step-like function:

$$
U_{a n}(z, r, \theta) \sim U_{0_{a n}} \cdot \Theta(|r-D|-(d / 2)) \cdot \Theta\left(|z|-L_{c} / 2\right),
$$

where $\Theta$ is Heavyside step-function, $D$ is radius of container, $d$ is a thickness of walls, $L_{c}$ is a length of container in $z$-direction, the potential wall $U_{0_{a n}} \rightarrow \infty$ is assumed to be much higher then chemical potential $\mu$. For trapped quantum gases the similar multiply connected potential configurations with a smooth penetrable walls are used. For example the toroidal trap inside a tightly focused $\left(F / D_{0} \cong 1\right.$, fig.1b) Laguerre-Gaussian (LG) beam forms the following trapping potential $U_{\text {tor }}$ in LG beam waist [3, 4]:

$$
U_{\text {tor }}(z, r, \theta) \sim \frac{U_{0} \cdot r^{2|\ell|}}{\left(1+z^{2} /\left(k^{2} D_{0}^{4}\right)\right)} \exp \left[-\frac{2 r^{2}}{D_{0}^{2}\left(1+z^{2} / k^{2} D_{0}^{4}\right)}\right],
$$

where $D_{0}$ is radius of LG beam, $k=2 \pi / \lambda, \lambda$ is the optical trapping wavelength, $\ell$ is azimuthal quantum number (topological charge) of LG, $F$ is the focal length of objective, $U_{0} \cong k_{B} T, T \sim 10^{-(3-6)} K$. This elongated torus has elliptical cross-section with $D_{0}^{2} / \lambda$ (Rayleigh range) width in $z$-direction and $\sim D_{0}$ width in $r$ (radial) direction. The current activity in toroidal traps is targeted to study Josephson phase dynamics and SQUID-like applications [3].

The periodic sequence of toroidal traps was also implemented as stationary interference pattern of counterpropagating LG beams (fig.1c):

$$
U_{\text {tor }_{P}}(z, r, \theta) \sim \frac{U_{0} \cdot r^{2|\ell|}}{\left(1+z^{2} /\left(k^{2} D_{0}^{4}\right)\right)} \exp \left[-\frac{2 r^{2}}{D_{0}^{2}\left(1+z^{2} / k^{2} D_{0}^{4}\right)}\right][1+\cos (2 k z)],
$$

\footnotetext{
*Electronic address: alexey.okulov@gmail.com URL: http://okulov-official.narod.ru
} 
where phase matching had been achieved by confocal parabolic mirrors [6] . This sequence of the $\lambda / 2$-separated toruses also has elliptical cross-section with $\lambda / 2$ width in $z$-direction and $\sim D_{0}$ width in radial direction [7].

In rotating toroidal containers (fig.1a,b,c) the circular flows of the liquid ${ }^{4} \mathrm{He}$ [1, 2] or degenerate quantum gas of a cold bosons in a toroidal trap [8 10] have a quantized circulation of the velocity $\vec{v}(\vec{r})$ along a closed contour $\Gamma$ embedded in a superfluid ensemble:

$$
\oint_{\Gamma} \vec{v}(\vec{r}) \cdot d \vec{r}=\frac{\hbar}{m} \oint_{\Gamma} \nabla \phi \cdot d \vec{r}=n \kappa, \kappa=\frac{2 \pi \hbar}{m},
$$

where $\kappa$ is a quantum of circulation, $n$ is integer, $\phi$ is the argument of the macroscopic wavefunction $\Psi$ [5]. When reference frame rotates with container with angular velocity $\Omega$ the circulation acquires the additional Sagnac term [11]:

$$
\oint_{\Gamma} \vec{v}(\vec{r}) \cdot d \vec{r}=\oint_{\Gamma}[\vec{v}(\vec{r})-\vec{\Omega} \times \vec{r}] \cdot d \vec{r}=n \kappa-2 \vec{\Omega} \cdot \vec{S}
$$

where $\vec{S}$ is area enclosed by contour $\Gamma$. Hence the phase of wavefunction is shifted by $\delta \phi_{S F}=(\mathrm{m} / \hbar) 2 \Omega \cdot \vec{S}$. Taking into account de Broglie wavelength $\lambda_{B}=2 \pi \hbar /(m|\vec{v}|)$ of massive particle moving with speed $|\vec{v}|$ the similarity between Sagnac shifts for massless photons $\delta \phi_{\text {phot }}$ [11, 12] and matter waves becomes evident.The numerical comparison shows that of matter wave Sagnac interferometer has better accuracy:

$$
\delta \phi_{S F}=\frac{4 \pi \vec{\Omega} \cdot \vec{S}}{\lambda_{B}|\vec{v}|}, \delta \phi_{p h o t}=\frac{4 \pi \vec{\Omega} \cdot \vec{S}_{p h o t}}{\lambda c}, \frac{\delta \phi_{S F}}{\delta \phi_{p h o t}}=\frac{\lambda m c|\vec{S}|}{h\left|\vec{S}_{p h o t}\right|}=\frac{m c^{2}|\vec{S}|}{h \nu\left|\vec{S}_{p h o t}\right|} \sim 10^{10} \frac{|\vec{S}|}{\left|\vec{S}_{p h o t}\right|}
$$

because of smallness of photon's energy $h \nu=h \lambda / c \sim 1 \mathrm{eV}$ compared to the rest mass of a typical atom. This advantage of the matter waves is a somewhat diminished by a substantially larger area $\left|\vec{S}_{\text {phot }}\right|$ enclosed by optical fibers compared to atomic waveguides $|\vec{S}|[11]$. This motivates further studies of atomic interference and search of the novel trapping geometries.

The aim of this article is to consider the influence of the reference frame rotations $\Omega$ on a superfluid confined by helical waveguide aligned along rotation axis $z$ [13, 14]. The interference of the counter propagating phase-conjugated Laguerre-Gaussian beams (LG) [15] carrying orbital angular momentum (OAM) [16] gives the following rotating potential profile for the red-detuned alkali atoms (fig.1d):

$$
U_{o p t}(z, r, \theta, t) \sim \frac{U_{0} \cdot r^{2|\ell|}}{\left(1+z^{2} /\left(k^{2} D_{0}^{4}\right)\right)} \exp \left[-\frac{2 r^{2}}{D_{0}^{2}\left(1+z^{2} / k^{2} D_{0}^{4}\right)}\right][1+\cos (2 k z+2 \ell \theta+\delta \omega t)],
$$

where the cylindrical coordinates $z, r, \theta$ are used, $D_{0}$ is the radius of LG [17], $\ell$ is vorticity, $k=2 \pi / \lambda$ is wavenumber, $\delta \omega$ is angular Doppler shift [18, 19] induced by rotation of the reference frame or emulated by rotation of Dove prism in a phase conjugated setup (fig.2) [20]. Transformation to the reference frame rotating synchronously with angular velocity $|\vec{\Omega}|=\Omega=\delta \omega / 2 \ell$ with trapping helix leads to the time-dependent Gross-Pitaevskii equation (GPE) [5, 14, 21]:

$$
i \hbar \frac{\partial \Psi}{\partial t}=-\frac{\hbar^{2}}{2 m} \Delta \Psi+\tilde{U}_{o p t}(z, r, \theta) \Psi+g|\Psi|^{2} \Psi-\Omega \hat{L}_{z} \Psi,
$$

where the stationary wavefunctions for the superfluid ensemble $\Psi=\Phi(z, r, \theta) \exp (-i \mu t / \hbar)$ are given by:

$$
\mu \Phi=-\frac{\hbar^{2}}{2 m} \Delta \Phi+\tilde{U}_{o p t}(z, r, \theta) \Phi+g|\Phi|^{2} \Phi+\Omega i \hbar \frac{\partial \Phi}{\partial \theta},
$$

where $\tilde{U}_{\text {opt }}(z, r, \theta)$ is time-independent optical potential. We evaluate linear and angular momenta of the superfluid ensemble in a helical container [14] and discuss the possibilities of rotations detection with this geometry. Hereafter the reference frame where laboratory is in rest will be called "laboratory frame" while reference frame collocated with viscous (classical) liquid completely trapped by rotating helical waveguide will be named "observer frame". Noteworthy the velocity vector $\vec{V}$ of "observer" with respect to "lab frame" has two components [22]: the azymuthal velocity $\vec{V}_{\theta}=\vec{\Omega} \times \vec{r}$ stands for helix rotation around LG propagation axis $\vec{z}$ while helix pitch velocity $\vec{V}_{z}=(\vec{z} / z) \delta \omega / 2 k$ is responsible for wavetrain translation along $\vec{z}$. 


\section{TWISTED WAVETRAINS IN ROTATING FRAME}

In order to reveal the basic features of helical confinement let us decompose the potential in the time-dependent GPE [5, 14] taking into account the paraxiality of laser eigenmodes. Namely the spatial scales in descending order are $z_{R}>>D_{0}>>\lambda / 2$, where $\lambda / 2 \sim 5-0.5 \mu m, D_{0} \cong 10-50 \mu m$ and $z_{R} \cong D_{0}^{2} / \lambda$ (Rayleigh range). Thus the potential $\tilde{U}_{\text {opt }}$ can be expanded in a Taylor series by small parameter $z \lambda /\left(D_{0}^{2}\right)$ (inverse Fresnel number):

$$
\begin{array}{r}
i \hbar \frac{\partial \Psi}{\partial t}=-\frac{\hbar^{2}}{2 m}\left[\frac{\partial}{\partial z^{2}}+\frac{\partial}{r \partial r} r \frac{\partial}{\partial r}+\frac{\partial^{2}}{r^{2} \partial \theta^{2}}\right] \cdot \Psi+g|\Psi|^{2} \Psi-\Omega \hat{L}_{z} \Psi+ \\
\tilde{U}_{o p t}(0) \cdot[1+\cos (2 k z+2 \ell \theta)] \cdot\left[\frac{m \omega_{z}^{2} z^{2}}{2 \cdot U_{o p t}(0)}+1\right] \cdot \frac{r^{2|\ell|}}{D_{0}^{2|\ell|}} \cdot \exp \left[-\frac{r^{2}}{D_{0}^{2}}\right] \cdot \Psi
\end{array}
$$

This decomposition is valid due to relatively slow diffractive spread of helical waveguide during motion through LGbeam waist from $z=0$ point. Consider first the matter wavetrain $\Psi$ localized within Rayleigh range $z<<z_{R}$. The atomic waveguide is not expanded here, hence GPE is as follows:

$$
\begin{array}{r}
i \hbar \frac{\partial \Psi}{\partial t}=-\frac{\hbar^{2}}{2 m}\left[\frac{\partial}{\partial z^{2}}+\frac{\partial}{r \partial r} r \frac{\partial}{\partial r}+\frac{\partial^{2}}{r^{2} \partial \theta^{2}}\right] \cdot \Psi+g|\Psi|^{2} \Psi-\Omega \hat{L}_{z} \Psi+ \\
\tilde{U}_{o p t}(0) \cdot[1+\cos (2 k z+2 \ell \theta)] \cdot \frac{r^{2|\ell|}}{D_{0}^{2|\ell|}} \cdot \exp \left[-\frac{r^{2}}{D_{0}^{2}}\right] \cdot \Psi
\end{array}
$$

The variational anzatz $\Psi_{h}(z, r, \theta, t)$ for the order parameter [23] in a form of the superposition of the two phaseconjugated matter wave vortices [14] is written for helical trap within LG-beam waist $\left(|z|<<z_{R}\right)$ as:

$$
\begin{array}{r}
\Psi_{h}(z, r, \theta, t)=\Psi_{\ell}(z, r, \theta, t)+\Psi_{-\ell}(z, r, \theta, t) \cong \\
\Psi_{ \pm \ell}(z=0) \cdot\left(r / D_{0}\right)^{|\ell|} \exp \left[-r^{2} / D_{0}^{2}\right] \cdot \exp \left[-\left[z-z_{1}(t)\right]^{2} / Z_{0}^{2}\right] \times \\
\left\{\exp \left[-i \mu_{f} t / \hbar+i k z+i \ell \theta\right]+\exp \left[-i \mu_{b} t / \hbar-i k z-i \ell \theta\right]\right\},
\end{array}
$$

where $Z_{0}$ is a longitudinal size of the matter wavetrain, $\mu_{f}, \mu_{b}$ are the chemical potentials of the "partial" wavefunctions $\Psi_{ \pm \ell}$. The density $\rho$ of this atomic wavetrain has a helical shape $\rho(z, r, \theta, t)=\left|\Psi_{h}\right|^{2}$ and this helix rotates with angular velocity $\Omega=\left(\mu_{f}-\mu_{b}\right) /(\hbar \cdot 2 \ell)$. The qualitative analysis based on anzatz $\Psi_{h}$ shows that due to the absence of friction the center of mass of superfluid ensemble remains in rest in laboratory frame. But in "observer frame" the center of mass will move along straight line $z_{1}(t)= \pm V_{B E C} \cdot t=(\Omega \ell / k) \cdot t=(\delta \omega / 2 k) \cdot t$, where $\delta \omega / 2 k$ is the group velocity of the wavetrain (fig.3A). The direction of this rectilinear motion is controlled by a product of the topological charge of trapping LG beams $\ell$ (the handedness of interference pattern) and projection of the vector of angular velocity $\vec{\Omega}$ of "observer frame" $\Omega$ on $z$ axis. Thus rotating observer will see translational motion of the center of mass of twisted wavetrain in the absence of any externally applied force. Noteworthy the order parameter $\Psi_{h}$ is a solution of the time dependent GPE (eq12) which describes purely condensed bosonic ensemble in the absence of thermal background.

Outside the beam waist $\left(|z| \geq z_{R}\right)$ the GPE with expanding trapping potential ought to be used (eq11).The variational anzatz for $\left|\Psi_{h}\right\rangle$ is also a superposition [14]:

$$
\begin{gathered}
\Psi_{h}(z, r, \theta, t)=\Psi_{\ell}(z, r, \theta, t)+\Psi_{-\ell}(z, r, \theta, t) \cong \\
\Psi_{ \pm \ell}(z=0) \cdot\left(r / D_{0}\right)^{|\ell|} \exp \left[-\frac{r^{2}}{D_{0}^{2}\left(1+i z / z_{R}\right)}\right] \cdot \exp \left[-\left[z-z_{2}(t)\right]^{2} / Z_{0}^{2}\right] \times \\
\left\{\exp \left[-\frac{i \mu_{f} t}{\hbar}+i k z+i \ell \theta\right]+\exp \left[-\frac{i \mu_{b} t}{\hbar}-i k z-i \ell \theta\right]\right\} \cdot\left(1+i z / z_{R}\right)^{-1} .
\end{gathered}
$$

Because red-detuned solutions are considered, the $\mid \Psi_{h}>$ is attracted by electrostatic potential $U_{\text {opt }}$ towards a beam waist $|z| \leq z_{R}$ and wavetrain is reflected from low optical intensity regions $|z|>z_{R}$ towards the center of trap. Thus center of mass oscillates with effective equation of motion:

$$
\ddot{z}_{2}(t)+\gamma_{Z} \cdot \dot{z}_{2}(t)+\left[\omega_{Z} /\left(1+2 \pi D_{0} / \lambda\right)\right]^{2} z_{2}(t)=F_{0} \cos (\Omega t)
$$

where $\omega_{z} \cong \hbar /\left(m z_{R}^{2}\right)$ is a frequency of oscillations in a "longitudinal" well, $F_{0}$ is the averaged amplitude of "kicks", caused by reflections from trap boundaries located near $z \sim z_{R}, \gamma_{Z}$ is phenomenological damping constant. The 
dissipation which is absent in initial GPE (eq11) appears in this model (eq 15) as phenomenological constant, arising because of leakage of atoms through potential barrier. The observer rotating with trap will see the onset of wavetrain oscillations (fig.3b) without any externally applied force. Consequently the helical waveguide for neutral atoms will transform rotation of lab frame into translational motion of the helical matter wave.

\section{ANGULAR MOMENTUM AND TRANSLATIONS OF SUPERFLUID IN ROTATING HELICAL PIPE}

It is instructive to evaluate exactly the macroscopic observables using variational wavefunction $\mid \Psi_{h}>$ (eqs 13 14). Because GPE (eqs 11, 12) gives coherent mean field wavefunction $\Psi$, the expectation values of linear $<\hat{P}_{z}>$ and angular momenta $\left\langle\hat{L}_{z}>\right.$ may be obtained by averaging over macroscopic wavefunction [9, 24]:

$$
<P_{z}>_{h}=<\Psi_{h}\left|-i \hbar \frac{\partial}{\partial z}\right| \Psi_{h}>=N \hbar\left(k_{f}-k_{b}\right)
$$

taking into account the mutual orientation of the optical angular momenta of LG beams in a trap

$$
<L_{z}>_{h}=<\Psi_{h}\left|-i \hbar \frac{\partial}{\partial \theta}\right| \Psi_{h}>=N \ell \hbar(1 \mp 1)
$$

where $N$ is the total number of bosons in a trap, the upper sign stands for the phase-conjugated LG-beams, i.e. for helical trap (fig.1d) [7, 14], while the bottom sign stands for sequence of toroidal traps aligned along the propagation axis $z$ (fig.1c). As it easily seen from (eq 16) the ensemble moves along $z$ with momentum $\hbar\left(k_{f}-k_{b}\right)$ per particle hence velocity of matter wave translation recorded by observer is $\Omega /\left(k_{f}+k_{b}\right)$. The angular momentum proves to be zero not only in observer frame but in a lab frame as well due to disappearance of the moment of inertia for rotating superfluid [5].

There exists a remarkable difference between proposed configuration (fig.2) and previously reported Sagnac matter wave interferometers [11] based on toroidal traps [3], matter wave grating interferometers 25] and rotating lowdimensional traps where circulation of velocities around closed contour $\Gamma$ is essential [26 28]. The circulation is proportional to the angular momentum of a classical particle. In a simplest case of circular rotation in a plane perpendicular to $z$ axis the angular momentum equals to it's projection $L_{z}=m|\vec{v}| r$. The quantization of the angular momentum leads to discrete expectation values of operator $\hat{L}_{z}<\Psi_{n}|-i \hbar \partial / \partial \theta| \Psi_{n}>= \pm n \hbar[24$. The quantum ensemble in a state $\Psi$ with zero circulation or zero angular momentum $n=0$ does not feel the rotation of container and may be referred to as an absolute reference frame. This is analog of the Meissner effect [9] had been confirmed experimentally both for the liquid helium in annular [1] and for the trapped alkali gases in rotating toroidal traps.

Nevertheless zero angular momentum state becomes thermodynamically unstable when the speed of angular motion $\Omega$ of container is increased above a certain critical value [5, 9]. In this case the Feynman criterion for vortex appearance is applicable where critical velocity $V_{c_{F}}$ is due to Bohr-Sommerfeld quantization condition around vortex core giving rotational flows with quantized angular momentum $n \hbar$ :

$$
\oint_{\Gamma} \vec{v} \cdot d \vec{r}=\ell \hbar / m, V_{c_{F}}=n \frac{\hbar}{m R} \ln \left(\frac{R}{R_{c}}\right) .
$$

The values of critical velocity in this multiply connected geometry are smaller than those predicted by Landau criterion for superfluidity where critical velocity in homogeneous condensate is a tangent to dispersion curve near roton's minimum $V_{c_{L}}=\epsilon(\vec{p}) /|\vec{p}|[2]$. The same holds for modified Landau criterion proposed for the rotating helical flow [14] in the form $\Omega_{c}=D_{0}^{-1} \epsilon(\vec{p}) /|\vec{p}|$ which correspond to excitation of rotons via centrifugal force and $\left|\vec{V}_{\theta}\right|=\Omega_{c} D_{0}$ is a tangential component of trapping helix velocity.

The angular momentum transfer from rotating environment to superfliud is facilitated by asymmetrical form of container [1] or externally imposed ponderomotive optical lattice potential but the actual underlying mechanism includes interaction of quantum (superfliud) and classical (normal) components of the ensemble [5, 8]. This interaction leads to formation of unbounded vortices and vortex lattices [29, 30]. For the sufficiently fast rotations when $\Omega$ becomes comparable to the classical transverse oscillation frequency $\omega_{\perp}$ the lowest Landau level (LLL) state appears and this is accompanied by solitons formation [28]. Noteworthy the vortex lattices with also demonstrate a certain independence from external rotations imposed by revolving square optical lattices [27]. 


\section{ROTATION OF HELICAL POTENTIAL VIA ANGULAR DOPPLER EFFECT}

Experimentally the rotation of the optical interference pattern [20, 29] has a definite instrumental advantages over mechanical rotation [1]. The essential features of helical tweezer traps realized in [15] are due to apparent topological difference of toroidal and helicoidal interference patterns [13](fig.2). The toroidal pattern appears when colliding photons have a parallel OAM's while helicoidal pattern arises for antiparallel photon's OAM's reflected from wavefront reversing mirrors (PCM) 7]. In contrast to the conventional mirrors the PCM are essentially anisotropic optical elements [7, 22, 31, 32]. Due to the internal helical structures, e.g. acoustical vortices in SBS mirrors, the PC mirror alters the angular momentum of the incident beam. Thus because the linear momentum $\vec{P}$ is altered too, the mutual orientation of $\vec{P}$ and $\vec{L}$ is conserved and the topological charge $\ell$ is not changed by an ideal PC mirror. This may be also a linear optical loop setup 12 provided the even number of reflections per a single loop roundtrip or the nonlinear optical PC retroreflector [7, 15].

The alternation of the orbital angular momentum in PC mirror is accompanied by conservation identities [20] for the frequencies of the incident and transmitted photons and the projection of the angular momenta $L_{z}$ on $z$-axis. When Dove prism rotates with the angular velocity $\vec{\Omega}$ we have:

$$
\begin{gathered}
I_{z z} \cdot \Omega+L_{z}=I_{z z} \cdot \Omega^{\prime}+L_{z}^{\prime} \\
\hbar \omega_{f}+\frac{I_{z z} \Omega^{2}}{2}=\hbar \omega^{\prime}+\frac{I_{z z} \Omega^{\prime 2}}{2},
\end{gathered}
$$

where left hand sides of this system correspond to the incident photon and the right hand sides correspond to the transmitted one, $I_{z z}$ is the moment of inertia around $z$-axis. The difference of the angular velocities of the prism before and after the photon passage is due to reversal of OAM projection from $L_{z}= \pm \ell \hbar$ to $L_{z}^{\prime}=\mp \ell \hbar$ eq. (19) :

$$
\Omega-\Omega^{\prime}=-\frac{2 \ell \cdot \hbar}{I_{z z}}
$$

From this follows that co-rotation increases the angular velocity of prism. Indeed the energy is transferred to the prism by the optical torque $|\vec{T}|=2 \ell \cdot P / \omega_{f}$, where $P$ is total power carried by LG [17]. As a result Doppler frequency shift for the photon $\omega^{\prime}-\omega_{f}$ is negative in this case:

$$
\delta \omega=\omega^{\prime}-\omega_{f}=\frac{I_{z z}}{2 \hbar}\left(\Omega-\Omega^{\prime}\right)\left(\Omega^{\prime}+\Omega\right)=-2 \ell \cdot \Omega-\frac{2 \ell \cdot \hbar}{I_{z z}} .
$$

The else consequence of angular momentum conservation is that reversal of angular velocity of the prism $\vec{\Omega}$ alters the angular Doppler shift sign $\delta \omega$ and helical trapping potential $\tilde{U}_{\text {opt }}(z, r, \theta, t)$ rotates in opposite direction. The remarkable feature of this phase-conjugating laser interferometer technique [20, 33] is that angular velocity of interference pattern $\Omega=\delta \omega / 2 \ell$ alters when vorticity of $L G$ beam $\ell$ is altered (fig.2).

\section{CONCLUSIONS}

The properties of superfluid ensemble in helical trap placed in rotating environment were analysed. For ultracold bosons $\left(10^{-6} \mathbf{K}\right)$ trapped in vacuum by helical optical interference pattern the Gross-Pitaevskii mean field theory was applied. The variational approach indicates the possibility of detection of the slow rotations of observer by means of measurement of mechanical momentum of atomic cloud. Namely the rotating observer will detect the appearence of the translational rectilinear motion and oscillations of matter wavetrain near equilibrium position of the trap without any externally applied force. The orbital angular momentum of trapping beams [18, 34] and rotational Doppler shift [19, 20] are the key components for the proposed experiment.

Hopefully the GPE-based model considered above is qualitavely applicable to the flow of liquid ${ }^{4} \mathrm{He}$ at $\mathbf{T}_{\lambda}<\mathbf{2 . 1 7 ^ { \circ } \mathbf { K }}$ temperatures through a $\mu m$-size capillary [35-37]. In particular the $\mu m$-size helical pipe filled by ${ }^{4} \mathrm{He}$ superfluid and placed to rotating container is expected to eject quantum fluid outwards the pipe with translational speed $V_{z}=\Omega \ell / k$ defined by angular frequency of container $\Omega$, the pitch of the helix $\lambda=k / 2 \pi$ and winding number $\ell$. The handedness of the helical pipe $\pm \ell$ defines the direction of superfluid translation along positive or negative direction of $\vec{z}$ axis. Apparently the visibility of effect should be much better for the temperatures much below $\lambda$-point because of higher density of the condensate fraction $\rho_{s} \rightarrow \rho$.

Qualitative picture is expected to be exactly opposite for classical fluid. In a frame collocated with rotating observer the classical fluid will stay in rest because of nonzero viscosity. In a rest (laboratory) frame the classical fluid will be 
pushed lengthways $z$-axis by rotating helical pipe. Thus the classical flow is controlled dynamically, by Navier-Stockes equation, i.e. by Newtonian forces and viscosity. On the contrary the quantum flow in rotating helical pipe is due to the purely kinematical reasons. In both cases the direction of translational velocity is defined by a sign of $\ell \cdot \Omega$ product.

[1] G. Hess and W. Fairbank,Phys.Rev.Lett., 19, 216 (1967).

[2] R.P.Feynman,"Statistical mechanics", Ch.11,(1972) Reading, Massachusetts.

[3] A. Ramanathan, K. C. Wright, S. R. Muniz, M. Zelan, W. T. Hill, III, C. J. Lobb, K. Helmerson, W. D. Phillips, and G. K. Campbell, Phys.Rev.Lett. 106, 130401 (2011).

[4] E. M. Wright, J. Arlt, and K. Dholakia, Phys.Rev.A 63, 013608 (2002).

[5] F. Dalfovo, S.Giorgini, S.Stringari, L.P.Pitaevskii, Rev.Mod.Phys.71,463(1999).

[6] T. Puppe, I. Schuster, A. Grothe, A. Kubanek, K. Murr, P.W.H. Pinkse, and G. Rempe, Phys.Rev.Lett., 99, 013002 (2007).

[7] A.Yu.Okulov, J.Phys.B., 41,101001 (2008).

[8] A.L.Fetter, Rev.Mod.Phys. 81, 647 (2009).

[9] A.J. Leggett, Rev.Mod.Phys. 73, 307-356 (2001).

[10] Yu. V. Bludov, and V.V.Konotop, Phys.Rev.A 75, 053614 (2007).

[11] M.O.Scully, M.S.Zubairy,"Quantum optics", Ch.4, (Cambridge University Press) (1997).

[12] A.Yu.Okulov, J. Opt. Soc. Am. B 27, 2424-2427 (2010).

[13] M.Bhattacharya, Opt.Commun. 279, 219 (2007).

[14] A.Yu.Okulov, Phys.Lett.A, 376, 650-655 (2012).

[15] M.Woerdemann, C.Alpmann and C.Denz, Opt. Express, 17, 22791(2009).

[16] L.Allen, M.W.Beijersbergen, R.J.C.Spreeuw and J.P.Woerdman, Phys.Rev.A, 45, 8185-8189 (1992)

[17] A. Bekshaev, M.Soskin and M. Vasnetsov, "Paraxial Light Beams with Angular Momentum", Nova Science(2008).

[18] Courtial J., Robertson D. A., Dholakia K., Allen L., Padgett M. J., Phys.Rev.Lett., 81,4828-4830(1998).

[19] J. Arlt, M. MacDonald, L. Paterson, W. Sibbett,K. Volke-Sepulveda and K. Dholakia, Opt. Express, 10 , 844 (2002).

[20] A.Yu.Okulov, J. Opt. Soc. Am. B 29, 714-718 (2012).

[21] J.Keeling and N. G. Berloff, Phys.Rev.Lett., 100,250401 (2008).

[22] A.Yu.Okulov, Phys.Lett.A, 374,4523-4527 (2010).

[23] B.A.Malomed, "Variational methods in nonlinear fiber optics and related fields", Progress in Optics,(E.Wolf, Editor: North Holland, Amsterdam ) 43, 69-191 (2002).

[24] L.D. Landau and E.M. Lifshitz,"Quantum Mechanics", Butterworth-Heinemann, Oxford(1976).

[25] A. Lenef, T.D. Hammond, E. T. Smith, M.S.Chapman, R.A.Rubenstein, and D.E.Pritchard,Phys.Rev.Lett., 78, 760-763 (1997).

[26] Y.V.Kartashov, B.A. Malomed, and L.Torner, Phys.Rev.A 75, 061602 (2007).

[27] H.Sakaguchi and B.A. Malomed, Phys.Rev.A 75, 013609 (2007).

[28] H.Sakaguchi and B.A. Malomed, Phys.Rev.A 7878, 063606 (2008).

[29] S.Tung, V.Schweikhard, and E.A.Cornell, Phys.Rev.Lett., 97,240402 (2006).

[30] S.Tung, V.Schweikhard, and E.A.Cornell, Phys.Rev.Lett., 99, 030401 (2007).

[31] A.Yu.Okulov, JETP Lett., 88, 631 (2008).

[32] A.Yu.Okulov, Phys.Rev.A , 80, 013837 (2009).

[33] N.G.Basov, I.G.Zubarev, A.B.Mironov, S.I.Mikhailov and A.Y.Okulov, JETP, 52, 847 (1980).

[34] J.Leach,M.J.Padgett,S.M.Barnett,S.Franke-Arnold, and J.Courtial, Phys.Rev.Lett., 88, 257901(2002).

[35] P.L.Kapitza, Nature, 141, 74 (1938).

[36] J.F.Allen, A.D.Misener, Nature, 141, 74 (1938).

[37] P.L.Kapitza, Phys.Rev., 60, 354 (1941). 

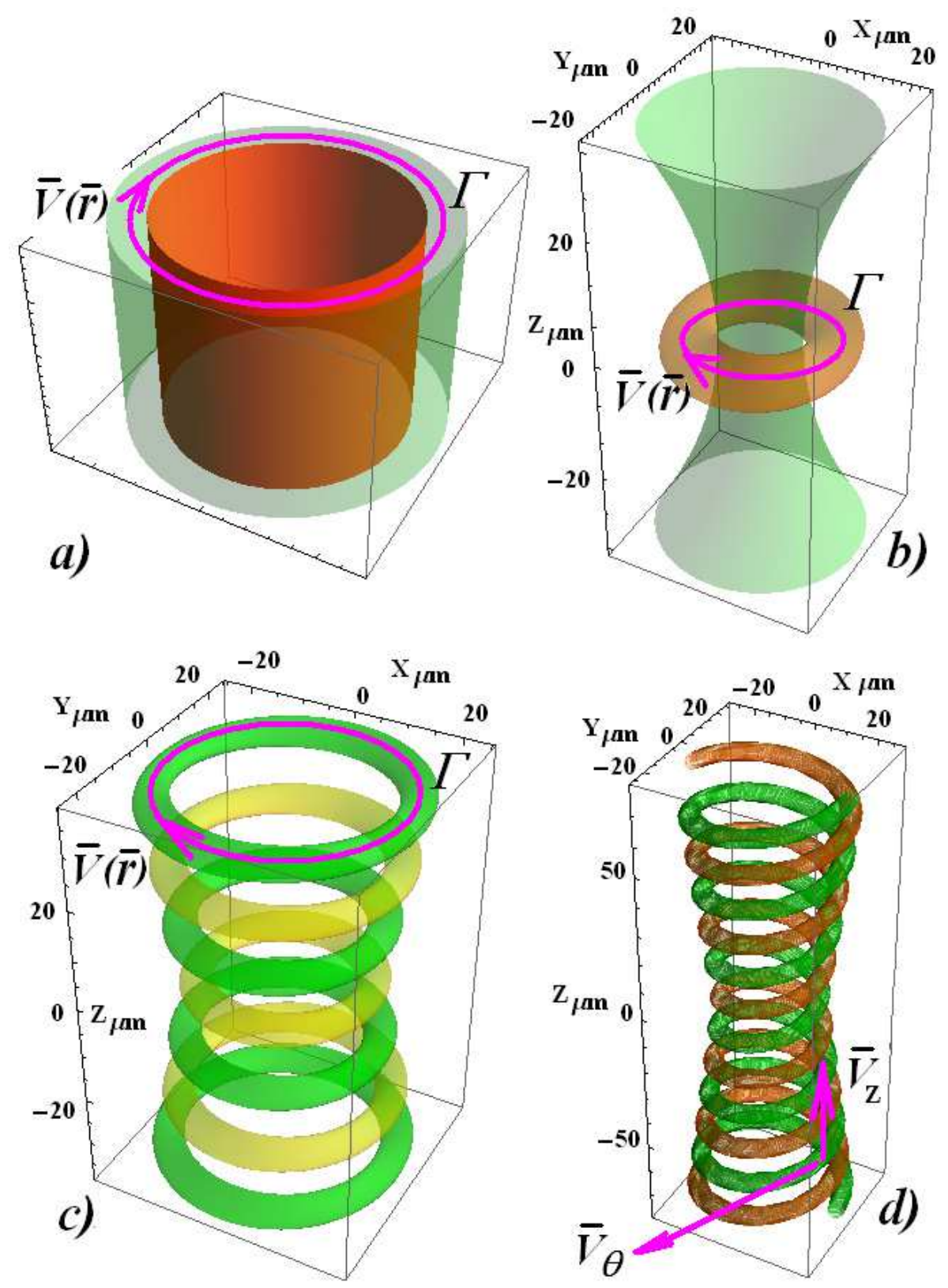

FIG. 1: Multiply connected potentials, $\vec{v}(\vec{r})$ is local velocity vector, $\Gamma$ - trajectory (contour) for calculating circulation : a) Annular cylinder in Hess-Fairbank angular momentum experiment [1], b) Toroidal potential in LG beam waist [3, 10] and in magnetic trap pierced by repulsive laser [3] , c) Sequence of toroidal wells separated by $\lambda / 2$ produced by two confocal $L G_{1,0}$ beams (with parallel OAM's) 6, 7, 15]. d) Helical interference pattern of the two phase-congugated $L G_{1,0}$ beams (with opposite OAM's) [6, 7, 14, 15] and velocity of observer $\vec{V}(\vec{r})=\vec{V}_{z}+\vec{V}_{\theta}$, which moves together with trapped classical liquid. 


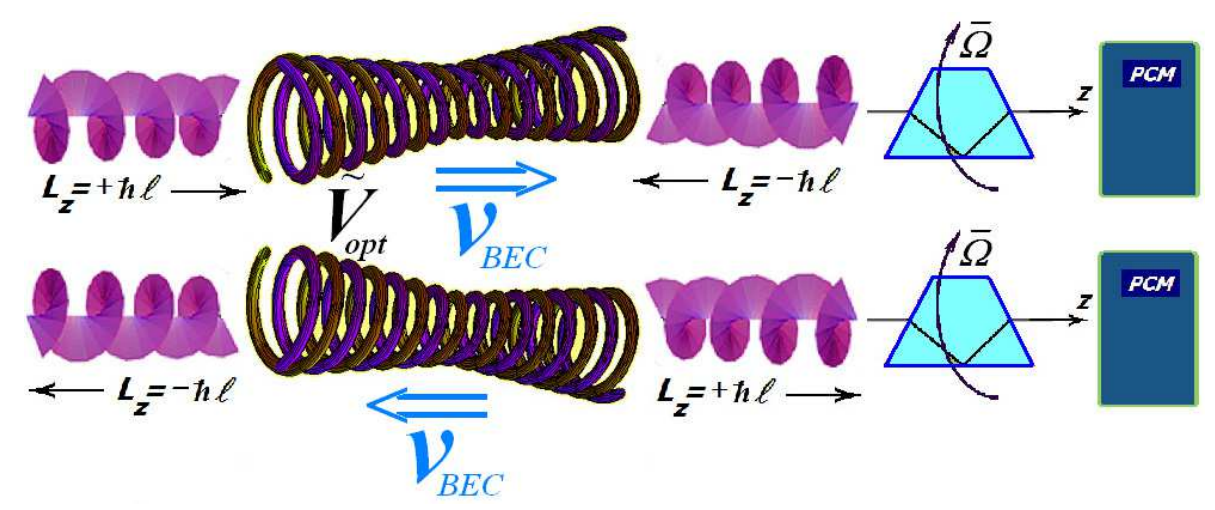

FIG. 2: Helical optical trapping potential $\tilde{U}_{\text {opt }}$ formed by two phase-conjugated optical vortices with opposite orbital angular momenta $\pm \hbar \ell$. Two mutual orientations of the angular momenta of vortices give the interference patterns of right handedness $(+\ell=1$ upper $)$ and of left handedness $(-\ell=1$ bottom) respectively. The rotation of interference pattern with angular frequency $\Omega$ is induced by rotating Dove prism located between trapping volume and phase-conjugating mirror PCM. This rotation is equivalent to rotation of the reference frame. The twisted BEC wavetrain in rotating helical atomic waveguide moves with a speed $V_{B E C}=\Omega \ell / k$ in a positive or a negative $z$ direction accordingly to the sign of $\Omega \ell$.
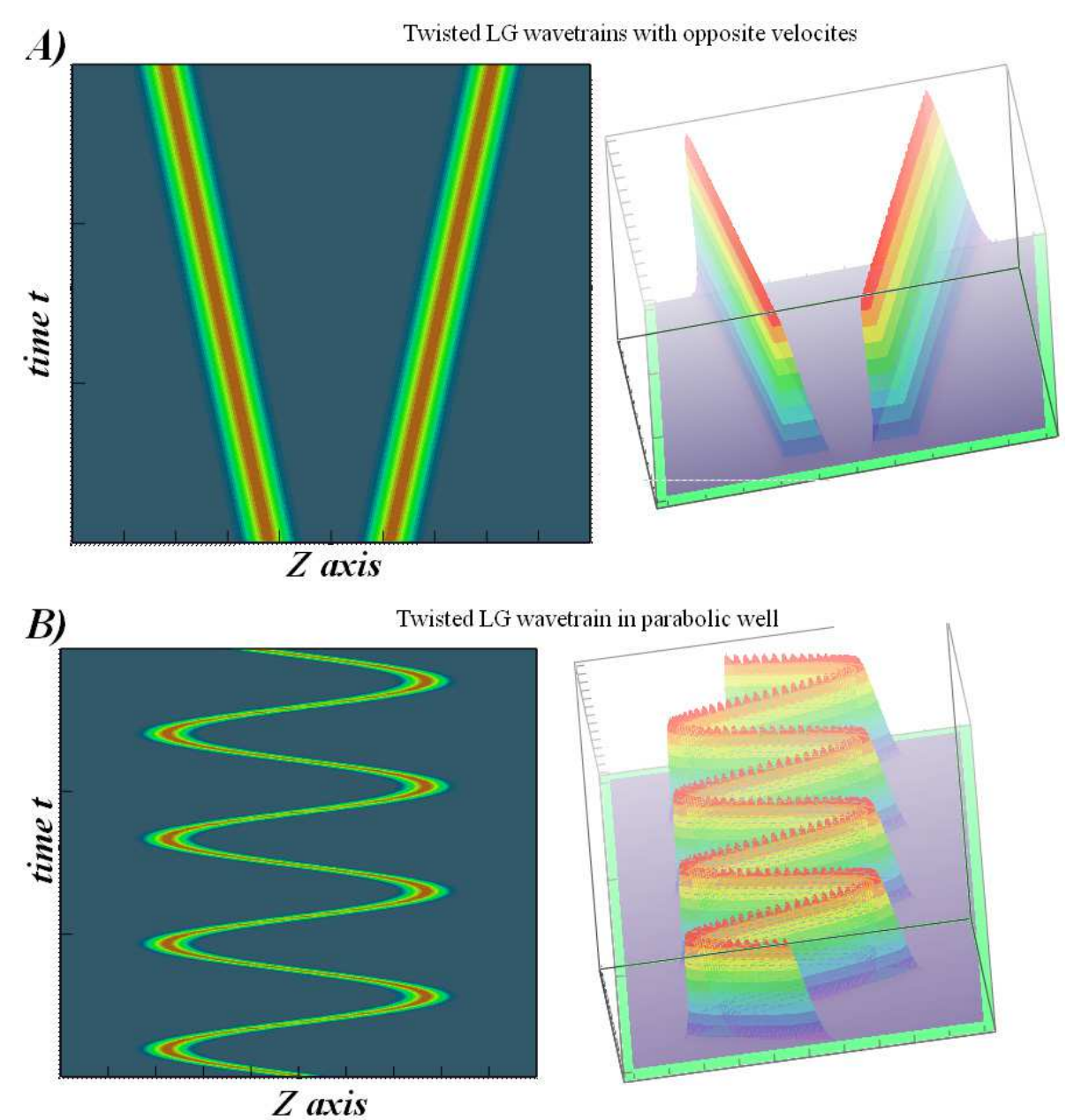

FIG. 3: Twisted matter wavetrain with Gaussian $z$-envelope in rotating helical atomic waveguide. a) In a waveguide with homegeneous ( $z$-independent) width of potential $U_{\text {opt }}$ the wavetrain will move with a constant speed $V_{B E C}= \pm \Omega \ell / k$ either positive or negative direction of $z$. b) In a waveguide with parabolic $z$-dependent potential $U_{\text {opt }}$ having width $D_{0}=D_{0}(1+$ $\left.z^{2} / z_{R}^{2}\right)$ ) the wavetrain will experience oscillations between reflection points [10]. 